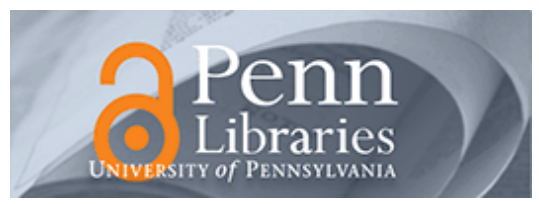

University of Pennsylvania

ScholarlyCommons

April 2004

\title{
Mechanical thresholds for initiation and persistence of pain following nerve root injury: mechanical and chemical contributions at injury
}

\author{
Beth A. Winkelstein \\ University of Pennsylvania, winkelst@seas.upenn.edu \\ Joyce A. DeLeo \\ Dartmouth-Hitchcock Medical Center
}

Follow this and additional works at: https://repository.upenn.edu/be_papers

\section{Recommended Citation}

Winkelstein, B. A., \& DeLeo, J. A. (2004). Mechanical thresholds for initiation and persistence of pain following nerve root injury: mechanical and chemical contributions at injury. Retrieved from

https://repository.upenn.edu/be_papers/27

Postprint version. Published in Journal of Biomechanical Engineering, Volume 126, Issue 2, April 2004, pages 258-263.

Publisher URL: http://dx.doi.org/10.1115/1.1695571

This paper is posted at ScholarlyCommons. https://repository.upenn.edu/be_papers/27

For more information, please contact repository@pobox.upenn.edu. 


\title{
Mechanical thresholds for initiation and persistence of pain following nerve root injury: mechanical and chemical contributions at injury
}

\author{
Abstract \\ There is much evidence supporting the hypothesis that magnitude of nerve root mechanical injury affects \\ the nature of the physiological responses which can contribute to pain in lumbar radiculopathy. \\ Specifically, injury magnitude has been shown to modulate behavioral hypersensitivity responses in \\ animal models of radiculopathy. However, no study has determined the mechanical deformation \\ thresholds for initiation and maintenance of the behavioral sensitivity in these models. Therefore, it was \\ the purpose of this study to quantify the effects of mechanical and chemical contributions at injury on \\ behavioral outcomes and to determine mechanical thresholds for pain onset and persistence. Male \\ Holtzman rats received either a silk or chromic gut ligation of the L5 nerve roots, a sham exposure of the \\ nerve roots, or a chromic exposure in which no mechanical deformation was applied but chromic gut \\ material was placed on the roots. Using image analysis, nerve root radial strains were estimated at the \\ time of injury. Behavioral hypersensitivity was assessed by measuring mechanical allodynia continuously \\ throughout the study. Chromic gut ligations produced allodynia responses for nerve root strains at two- \\ thirds of the magnitudes of those strains which produced the corresponding behaviors for silk ligation. \\ Thresholds for nerve root compression producing the onset (8.4\%) and persistence of pain (17.4-22.2\%) \\ were determined for silk ligation in this lumbar radiculopathy model. Such mechanical thresholds for \\ behavioral sensitivity in a painful radiculopathy model begin to provide biomechanical data which may \\ have utility in broader experimental and computational models for relating injury biomechanics and \\ physiologic responses of pain.
}

\section{Keywords}

radiculopathy, nociception, nerve root, biomechanics, strain

\section{Comments}

Postprint version. Published in Journal of Biomechanical Engineering, Volume 126, Issue 2, April 2004, pages 258-263.

Publisher URL: http://dx.doi.org/10.1115/1.1695571 


\title{
MECHANICAL THRESHOLDS FOR INITIATION \& PERSISTENCE OF PAIN FOLLOWING NERVE ROOT INJURY: MECHANICAL \& CHEMICAL CONTRIBUTIONS AT INJURY
}

\author{
Beth A. Winkelstein, Ph.D. ${ }^{1}$ and Joyce A. DeLeo, Ph.D. ${ }^{2}$ \\ ${ }^{1}$ Department of Bioengineering \\ University of Pennsylvania \\ Philadelphia, PA 19104 \\ ${ }^{2}$ Departments of Anesthesiology \& Pharmacology \& Toxicology \\ Dartmouth-Hitchcock Medical Center \\ Lebanon, NH 03756
}

Corresponding Author \& Reprint Requests: Beth A. Winkelstein, Department of Bioengineering, University of Pennsylvania, 120 Hayden Hall, 3320 Smith Walk, Philadelphia, PA, 19104-6392, (215) 573-4589, Fax (215) 573-2071, winkelst@,seas.upenn.edu.

Keywords: radiculopathy, nociception, nerve root, biomechanics, strain 


\section{ABSTRACT}

There is much evidence supporting the hypothesis that magnitude of nerve root mechanical injury affects the nature of the physiological responses which can contribute to pain in lumbar radiculopathy. Specifically, injury magnitude has been shown to modulate behavioral hypersensitivity responses in animal models of radiculopathy. However, no study has determined the mechanical deformation thresholds for initiation and maintenance of the behavioral sensitivity in these models. Therefore, it was the purpose of this study to quantify the effects of mechanical and chemical contributions at injury on behavioral outcomes and to determine mechanical thresholds for pain onset and persistence. Male Holtzman rats received either a silk or chromic gut ligation of the L5 nerve roots, a sham exposure of the nerve roots, or a chromic exposure in which no mechanical deformation was applied but chromic gut material was placed on the roots. Using image analysis, nerve root radial strains were estimated at the time of injury. Behavioral hypersensitivity was assessed by measuring mechanical allodynia continuously throughout the study. Chromic gut ligations produced allodynia responses for nerve root strains at two-thirds of the magnitudes of those strains which produced the corresponding behaviors for silk ligation. Thresholds for nerve root compression producing the onset $(8.4 \%)$ and persistence of pain $(17.4-22.2 \%)$ were determined for silk ligation in this lumbar radiculopathy model. Such mechanical thresholds for behavioral sensitivity in a painful radiculopathy model begin to provide biomechanical data which may have utility in broader experimental and computational models for relating injury biomechanics and physiologic responses of pain. 


\section{INTRODUCTION}

Low back pain affects as many as two-thirds of the adult population in Western Society and has an estimated annual cost of $\$ 38-50$ billion [1-3]. Painful lumbar radiculopathy (LR) commonly results from mechanical compression of lumbar nerve roots, either by disc prolapse or spinal stenosis [4-7]. A complicated combination of neurologic, electrophysiological, biochemical, structural, and mechanical contributions producing persistent pain, likely act alone and in concert $[6,8,9]$. Ongoing research has focused on understanding each of these areas individually as they contribute to painful LR [10]. However, while the relationships between mechanical injury and pain responses are currently being delineated, no work to date has defined mechanical thresholds for pain-related behaviors in these models.

Experimental studies using lumbar nerve root compression have demonstrated relationships between tissue compression and behavioral hypersensitivity responses [11-15]. In these animal models, lumbar nerve root compression is produced by ligation, with or without chromic gut suture material providing a simultaneous chemical irritation due to disc herniation. Models of chronic pain have used chromic gut material to simulate the inflammatory effects of extruded disc material $[11,13]$ and these models have been previously validated for producing behavioral sensitivity which mimics that produced by placing disc material directly on nerve roots [16]. Differential persistent pain behaviors are produced depending on the nature of the insult. A typical behavioral response includes mechanical allodynia (an increased sensitivity to a non-noxious stimulus) and is observed in the affected hind paw [11]. In animal models, allodynia is often measured by the frequency of paw withdrawals elicited by stimulation with otherwise non-noxious von Frey filaments [11,17]. Allodynia is also a representative clinical sign observed in LR patients and provides a gauge of nociceptive responses. It has been shown 
quantitatively that the more severe the mechanical injury, the greater the resulting behavioral hypersensitivity $[10,11,14]$. This is consistent with graded electrophysiologic responses by injury intensity described by Pedowitz et al. [18], and suggests one possible mechanism by which differential clinical symptoms may be produced. However, no thresholds have been provided for mechanical loading which produces behavioral sensitivity in this context.

Therefore, it is the purpose of the present study to utilize in vivo imaging techniques to quantify the contributions of mechanical nerve root tissue compression in the presence and absence of a chemical insult. It is hypothesized that the presence of chromic gut at the time of injury produces more robust behavioral sensitivity responses and may lower the threshold for mechanical injury leading to pain. Using assessments of allodynia, mechanical thresholds for pain initiation and persistence are defined for nerve root mechanical compression in a radiculopathy model.

\section{METHODS}

Experiments were performed using male Holtzman rats, each weighing 225-275 grams at surgery. Animals were housed individually under USDA and AAALAC-approved conditions with a 12-12 hour light-dark cycle and free access to food and water. All experimental procedures were approved by the Dartmouth College Institutional Animal Care and Use Committee.

\section{Surgical Protocol}

Surgical procedures were performed under inhalation halothane anesthesia $(4 \%$ induction, $2 \%$ maintenance). Surgical methods for this LR model have been previously described [14,15]. Briefly, using an operating microscope (LFS 200, Carl Zeiss Inc.), a left 
hemilaminectomy was performed at L5. At the time of surgery, animals were divided into four groups: (1) silk ligations $(\mathrm{n}=18)$ in which the $\mathrm{L} 5$ dorsal and ventral nerve roots were tightly ligated using a single 6-0 silk suture, (2) chromic ligations $(\mathrm{n}=7)$ in which the L5 dorsal and ventral nerve roots were tightly ligated using a single 6-0 chromic gut suture, (3) shams $(n=4)$ in which the L5 roots were exposed only, and (4) chromic exposures $(n=2)$ in which the L5 nerve roots were exposed and 3 pieces of $1.5 \mathrm{~mm} \mathrm{6-0}$ chromic gut were placed on them, approximating the total length of chromic suture used in the chromic ligations. Following surgery, wounds were closed with 3-0 polyester suture and surgical staples. Animals were recovered in room air.

The operating scope was equipped with a digital camera (Model DP10, Olympus Optical Co.) (1024x1280 pixel resolution) to image neural tissue in vivo during surgery. At least two marks were made on the L5/L6 bony facet surfaces using acrylic black paint to provide positional data defining a local origin and orientation for each image of an animal's surgery (Figure 1). Images were acquired for initially undeformed and immediately post-ligation configurations of the nerve root. Animals were followed postoperatively for 14 days.

\section{Image and Strain Analysis}

In vivo methods for recording and estimating nerve root radial strains in this model have been previously described $[14,15]$. At the time of surgery, images were also acquired to provide geometric calibration. Using the bony marker locations and calibration images, each image for a given animal was transformed into the coordinate system defined by its initial reference image, allowing consistent definitions and comparison of geometric measurements. Bony landmarks were digitized using ImageTool Software (UTHSCSA, San Antonio, TX). These locations and the line defined by their endpoints were used to transform image orientations to the reference coordinate system. The nerve root boundary was digitized along its outer-most edges, providing 
a set of contours for the nerve root in its in vivo unligated and ligated geometries (Figure 1). For shams, contour sets consisted of two unligated geometries.

Cubic polynomials were fit to the digitized boundaries. To estimate radial strain, nerve roots were approximated as cylindrical with a circular cross-section and variable diameter along the length. The diameter was calculated from the two contour boundaries in the radial direction. Customized Fortran code determined the centerline and corresponding radius from each set of boundaries. Radial strain $\left(\varepsilon_{\mathrm{R}}\right)$ was calculated along the nerve root length using the initial unligated $\left(\mathrm{r}_{\mathrm{ref}}\right)$ measurements as reference [19], where:

$$
\varepsilon_{R}=\frac{r-r_{r e f}}{r_{r e f}} .
$$

Calculations were performed in the region of maximal compression. All digitization was performed by a single digitizer, blinded to all other measurements of this study.

\section{Behavioral Testing}

All animals undergoing surgery were evaluated for mechanical allodynia in the ipsilateral hind paw at days 1,3,5,7,10 and 14, postoperatively. Mechanical allodynia was measured as the number of hind paw withdrawals elicited by a defined non-noxious mechanical stimulus [20]. Animals were previously acclimated to the testing environment and tester. Baseline measurements were acquired for each animal prior to surgery. All behavioral testing was performed by the same tester, blinded to the surgical procedure. In each testing session, rats were subjected to three rounds of 10 tactile stimulations to the plantar surface of the hind paw using 2 and 12 gram von Frey filaments (Stoelting, Wood Dale, IL). 


\section{Statistical Analysis}

For the purpose of assessing changes in behavioral sensitivity (allodynia), the silk group was further divided according to the magnitude of applied strain: a low group $(\mathrm{n}=10)$ consisted of those ligations which were applied "as loosely as possible" and a tight group ( $\mathrm{n}=8)$ in which ligations were applied as tightly as possible. Likewise, the chromic group was similarly divided according to applied strain: low chromic $(n=3)$ and tight chromic $(n=4)$ groups. Comparisons of applied strains and the time-dependent allodynia curves between all groups were performed using a repeated analysis of variance (ANOVA), with significance at $\mathrm{p} \leq 0.05$. For animals undergoing ligations, correlations were performed between the magnitude of applied ligation strain and resulting total allodynia. Significance was defined at $\mathrm{p} \leq 0.05$.

\section{Mechanical Thresholds for Pain Behaviors}

Total allodynia was determined for each animal in the sham and silk ligation groups as the cumulative sum of the paw withdrawals (using 12 gram) postoperatively. For determining the threshold for pain initiation, the highest total allodynia measured for any of the sham animals (13 withdrawals) was used to define onset of a pain behavioral response. Each animal undergoing silk ligation was assigned a pain score of 1 or 0 based on having a total allodynia score: (1) above 13 or (0) equal to or below 13. Likewise, the mean total allodynia for the silk group ( $47.6 \pm 34.6$ withdrawals) was used to define a behavioral response exhibiting persistent

pain. In this case, a persistent pain score was assigned 1 for those responses above the mean and 0 for those below it. For each of these two definitions, total allodynia was plotted against the applied nerve root strain measured for each animal. Logistic regression was used to determine the $50^{\text {th }}$ and $95^{\text {th }}$ percentile threshold values for pain behaviors due to silk ligation [21].

\section{RESULTS}


All applied nerve root ligation strains were compressive. Nerve roots for rats used in this study have a mean diameter of $1.45 \pm 0.42 \mathrm{~mm}$. Mean errors in estimating in vivo radial strains using this technique for imaging, digitization, boundary curve fitting, and strain analysis have been previously reported as $0.9 \pm 0.9 \%$ [14]. This error is small compared to strains applied in this study. For all shams, the mean calculated strain from image analysis was $0.32 \pm 0.79 \%$, which is within the range of analysis errors, indicating no measured tissue injury. The mean applied strain for silk ligations was $19.7 \pm 10.6 \%$. This includes the low group with ligations applied as loosely as possible and the tight group with ligatures tightened to the nerve root. The mean applied strains in these two groups were different $(\mathrm{p}=0.001)$ and were $12.4 \pm 1.5 \%$ and $28.8 \pm 8.6 \%$ for the low and tight groups, respectively. In the chromic ligations, mean applied strain at ligation was $19.2 \pm 11.6 \%$, which was significantly different from the silk ligations $(\mathrm{p}=0.04)$. Likewise, the low chromic group had a mean applied strain of $8.8 \pm 9.6 \%$ which was significantly lower $(\mathrm{p}=0.028)$ than the tight chromic group mean strain of $27.1 \pm 4.5 \%$.

All animals undergoing nerve root ligation exhibited mechanical allodynia following injury (Figure 2). Despite significantly different injury strains between the two chromic groups, allodynia patterns were not significantly different. Therefore, given the lack of behavioral dependence on strain in the presence of chromic, the insults for the chromic groups were analyzed as one injury group. The typical response for allodynia was observed for the tight and chromic groups, with a robust initial response which was maintained with only minor decreases over time. This pattern of behavioral hypersensitivity was observed for both 2 and 12 gram stimulations (Figure 2, $2 \mathrm{gm}$ data not shown). Allodynia produced for the tight and chromic ligation injuries was elevated over sham, chromic exposure, and low for all testing ( $\mathrm{p}<0.0005)$. In contrast, while both the low and chromic exposure animals exhibited elevated allodynia 
compared to sham responses, these increases were only slight and not significant for either group or von Frey filament tested $(\mathrm{p}>0.136)$. Despite having significantly greater $(\mathrm{p}=0.001)$ applied tissue deformations, mechanical allodynia responses produced in the tight silk $(28.8 \pm 8.6 \%)$ and chromic ligation $(19.2 \pm 11.6 \%)$ groups were not different $(\mathrm{p}>0.243)$.

Correlations between injury magnitude and total allodynia, were also performed. Separate correlations were performed for silk and chromic ligations (Table 1). Total allodynia over 14 days following injury showed very weak correlation with applied mechanical compression in the presence of chromic gut material (Figure 3, Table 1); and were not significant. However, for the silk ligations, both 2 and 12 gram allodynia responses displayed significant correlation with the degree of applied compression (Figure 4, Table 2).

Pain threshold analysis was performed for silk ligation of the L5 nerve roots. Total postoperative allodynia for silk ranged from 3 to 105 withdrawals, with a median value of 27.5 withdrawals. The threshold for nerve root deformation initiating a pain response, while not necessarily required to maintain it, was determined using the greatest cumulative allodynia response of the sham animals (13 withdrawals). Allodynia responses greater than sham levels were observed in 13 ligated animals, while only 5 ligated animals had allodynia responses comparable to sham levels. Based on logistic regression of these responses compared with applied injury magnitude, it was determined that at a nerve root compression of $8.4 \%$, half of all animals will exhibit the onset of pain-associated behaviors. Likewise, persistent mechanical allodynia over the 14 days postoperatively was observed in 7 animals and was not maintained in 11 of the animals undergoing these silk ligations (Figure 5). The lowest applied strain producing persistent allodynia was observed at $17.4 \%$ and produced a total response of 60 paw 
withdrawals. For this analysis, the $50^{\text {th }}$ percentile strain of $20.8 \%$ produced behavioral hypersensitivity which was characteristic of persistent pain. Strain of $22.2 \%$ was predicted to produce persistent allodynia in this model for $95 \%$ of animals.

\section{DISCUSSION}

This study demonstrates that both the magnitude of mechanical tissue deformation (strain) and the chemical environment at nerve root injury play distinct roles in producing behavioral sensitivity associated with lumbar radiculopathy. While many in vivo investigations have suggested that persistent pain is modulated by both of these factors (mechanical \& chemical) $[5,11,17,22,23]$, no study has been performed which directly quantifies the injury severity magnitude and measures of nociception and pain. Moreover, while qualitative reports have implicated biomechanics in initiating physiologic responses with electrophysiologic, structural, immunologic and behavioral components, this study provides thresholds for mechanical tissue deformation which produce the onset and maintenance of persistent pain.

While this study is not the first to quantify mechanical nerve root injury severity in the context of physiologically relevant nociceptive responses for LR [5,6,11,14,15,18,22,24], it is the first report to determine specific thresholds for initiating and maintaining persistent behavioral hypersensitivity as measured by mechanical allodynia. Implementation of in vivo imaging techniques allows for precise quantification of tissue deformations applied via ligation. In the context of this previously well-characterized rodent model of lumbar radiculopathy, this work defines the $50^{\text {th }}$ and $95^{\text {th }}$ percentile thresholds for persistent pain in this model at $20.8 \%$ and $22.2 \%$, respectively. The methods of logistic regression used in this study apply a similar methodology as has been previously used to define mechanical thresholds for morphological and 
electrophysiological damage in axonal injury [21], further demonstrating a utility to such an approach for defining relationships between mechanics and neural injury. However, given the limitations in image analysis techniques used here, it may be more appropriate to suggest a range of strain values (17.4-22.2\%) within which this threshold for persistence may exist. It is important to note that while the strain estimation techniques employed in this study are limited by the one-dimensional nature of their measurements, they have a precision of approximately $1 \%$ [14]. In addition, this approach for quantifying applied radial strain is accurate to $1.5 \%$ strain when compared to direct measurements of applied nerve root compressive strains [25]. These error measurements further suggest that from a mechanical standpoint there may not be a distinct physiologic difference between the 20.8 and $22.2 \%$ threshold levels.

Comparing the mechanical tolerances for persistent sensitivity following nerve root impingement determined in this study to neural foramen occlusion values determined for human cadaveric and volunteer studies provides useful context for their interpretation. For example, lumbar foraminal space occlusion has been reported to be as high as $33 \%$ for sagittal bending [26,27]. Likewise, using magnetic resonance imaging, asymptomatic volunteers have demonstrated changes in foraminal cross-sectional area of as much as $23 \%$, with no neural impingement [28]. While these studies do not directly provide measures of nerve root deformations, they begin to provide a potential link to neural injury and suggest that such a mechanism for pain due to nerve root impingement is indeed possible in humans. In addition, while compressive strains are presented in this study for applied radial deformations, there is no direct measurement of the coupled strains along the long axis of the nerve root. While loading along the longitudinal direction of the nerve root may contribute to the mechanical injury, it is also likely that local tensile and shear loading at the ligation site may impose greater insults to 
neuronal projections. Indeed, these measurements were not made in this study, but are undoubtedly important for understanding the specific nature of painful nerve root injuries.

While this work suggests compressive tissue tolerances for eliciting mechanical allodynia for nerve root compression, they represent only one aspect of the nociceptive responses of pain, mechanical allodynia. For example, this work does not describe those thresholds for loading above which specific physiological response are initiated. The thresholds defined here do not indicate when production and/or release of spinal cytokines is elevated, when glial activation occurs, or when electrophysiologic changes occur. These physiologic responses have been shown to contribute to central sensitization and mechanical allodynia in models of persistent pain $[11,17,29,30]$. While tactile hypersensitivity is an important measure for pain since it relates directly to clinical symptoms reported by pain patients, it is likely a manifestation of many physiologic cascades which are interconnected [10]. Biomechanics at injury have a role in modulating behavioral responses and their associated central neuroimmune cascades. For example, in this radiculopathy model, spinal cytokine mRNA levels at day 7 are correlated with the magnitude of imposed tissue strain at injury [14], indicating mechanical modulation of one aspect of spinal neuroimmune changes. Similarly, using immunohistochemistry and qualitative assessment of injury severity as either "tight" or "loose," spinal expression of the proinflammatory cytokine IL-1 $\beta$ was found to be more intense for "tight" ligations which exhibited greater behavioral hypersensitivity [11].

This study shows behavioral sensitivity responses are modulated by both mechanical and chemical components at the time of injury (Figure 2). The findings of this study are consistent with other less quantitative approaches used in pain research $[5,11,17,22,23]$. Allodynia 
intensity is increased for greater nerve root strains in the absence of chromic material. However, the same degree of behavioral hypersensitivity is produced for ligations with chromic suture which have strain magnitudes two-thirds that of the tight silk ligations (Figure 2), suggesting that in the presence of inflammatory chromic gut, less tissue deformation is required to elicit tactile hypersensitivity. Also demonstrated in the silk ligation group, is that total allodynia is directly correlated with injury strain magnitude and changes accordingly (Figure 4). However, this relationship to applied tissue strain does not exist in the presence of chromic gut material (Figure 3). In fact, there is no detectable difference in allodynia produced for strains over a $30 \%$ range, suggesting that mechanical injury may play less of a significant role in behavioral hypersensitivity if chemical irritants are present, such as chromic gut material or a herniated nucleus pulposus. Such an experimental finding offers an explanation for why differential clinical outcomes are present for disc herniations with seemingly similar nerve root impingement. While this study suggests the role of chemical factors in influencing behavioral outcomes for mechanical injuries, it does not fully address the specific physiologic or injury mechanisms responsible for these changes. Continued efforts are needed to elucidate the specific mechanisms of persistent pain due to mechanical injury.

It should be noted that for this study, the number of animals in the chromic group was half that in the silk group. The range of applied strains in this group was smaller than that in the silk ligations. With greater numbers over a broader range of applied strains, stronger correlations might be observed for chromic behavioral hypersensitivity responses (Table 1). One reason for this limitation in applied strains comes from the fact that chromic gut suture material is much less flexible than silk suture of the same size, making it difficult to apply tighter ligations than those reported here with the chromic gut suture. Moreover, while the mechanical contribution at 
injury is quantified in this study, the chemical component is not, adding uncertainty to a quantitative understanding of the relationship between injury and behavioral outcomes. Of particular interest with clinical relevance, for chromic exposure in the absence of mechanical deformation, mechanical allodynia was not different from shams, suggesting mechanical injury may be a requisite for allodynia development in this model. This is in contrast to previously reported work producing postural and gait alterations for chromic gut placed near the sciatic nerve in a rat model of neuropathy [31].

Together, the findings of this study offer information which contributes to an evolving understanding which is necessary for effective prevention and treatment of spinal pain syndromes, especially in the context of mechanical loading and thresholds. The mechanical thresholds demonstrate that separate tolerances exist for the initiation and maintenance of behavioral sensitivity (allodynia) observed in this painful lumbar radiculopathy model. Continued efforts to quantify and control injury mechanics (both deformation and force) are necessary to fully understand the mechanical nature of this injury. This work indicates that the biomechanics and chemical environment of the injury event contribute to behavioral hypersensitivity. In a broader context, this study begins to provide quantitative data which offer potential utility in other biomechanical modeling applications where any link between mechanics and the physiology of pain is currently only inferential. 


\section{ACKNOWLEDGMENTS}

The authors would like to thank the following for grant/gift support: National Institute of Neurological Disorders and Stroke NS011161 (BAW); the National Institute of Arthritis and

Musculoskeletal and Skin Diseases AR44757 (JAD), AR47564 (BAW); Bristol-Myers Squibb/Zimmer Orthopaedic Foundation (JAD). 


\section{REFERENCES}

[1] Deyo, R. A., and Tsui-Wu, Y. J., 1987, “Descriptive Epidemiology of Low-Back Pain and its Related Medical Care in the United States," Spine, 12, pp. 264-268.

[2] Frymoyer, J., and Cats-Baril, W., 1991, “An Overview of the Incidences and Costs of Low Back Pain,” The Orthopedic Clinics of North America, 22, pp. 263-271.

[3] Frymoyer, J., and Durett, C., 1999, "The Economics of Spinal Disorders," The Adult Spine: Principles and Practice, Frymoyer, J. W., ed.,. Lippincott-Raven Publishers, Philadelphia.

[4] Hollingworth, W., Dixon, A. K., Todd, C. J., Bell, M. I., Antoun, N. M., Arafat, Q., Girling, S., Karia, K. R., and Laing, R. J., 1998, "Self Reported Health Status and Magnetic Resonance Imaging Findings in Patients with Low Back Pain,” European Spine Journal, 7, pp. 369-375.

[5] Olmarker, K., and Myers, R. R., 1998, "Pathogenesis of Sciatic Pain: Role of Herniated Nucleus Pulposus and Deformation of Spinal Nerve Root and Dorsal Root Ganglion," Pain, 78, pp. 99-105.

[6] Rydevik, B., Hasue, M., Wehling, P., 1996, "Etiology of Sciatic Pain and Mechanisms of Nerve Root Compression,” The Lumbar Spine, Wiesel, S.W., Weinstein, J.N., Herkowitz, H., Dvorak, J., Bell, G., eds., WB Saunders Co., Philadelphia, pp 123-141. 
[7] Vucetic, N., Astrand, P., Guntner, P., and Svensson, O., 1999, "Diagnosis and Prognosis in Lumbar Disc Herniation,” Clinical Orthopaedics \& Related Research, 361, pp. 116-122.

[8] Wall, P., and Melzack, R., 1994, Textbook of Pain, $3^{\text {rd }}$ Edition, London: Churchill Livingstone.

[9] Weinstein, J., and Gordon, S., 1996, Low Back Pain: A Scientific and Clinical Overview, Illinois: AAOS.

[10] DeLeo, J. A., and Winkelstein, B. A., 2002, "Physiology of Chronic Spinal Pain Syndromes: From Animal Models to Biomechanics,” Spine, 27, pp. 2526-2537.

[11] Hashizume, H., DeLeo, J. A., Colburn, R. W., and Weinstein, J. N., 2000, "Spinal Glial Activation and Cytokine Expression after Lumbar Root Injury in the Rat,” Spine, 25, pp. 1206-1217.

[12] Howe, J., Loesser, J., and Calvin, W., 1977, "Mechanosensitivity of Dorsal Root Ganglia and Chronically Injured Axons: A Physiological Basis for the Radicular Pain of Nerve Root Compression," Pain, 3, pp. 25-41. 
[13] Kawakami, M., Weinstein, J. N., Chatani, K., Spratt, K. F., Meller, S. T., and Gebhardt, G. F., 1994, "Experimental Lumbar Radiculopathy. Behavioral and Histologic Changes in a Model of Radicular Pain after Spinal Nerve Root Irritation with Chromic Gut Ligatures in the Rat,” Spine, 19, pp. 1795-1802.

[14] Winkelstein, B. A., Rutkowski, M. D., Weinstein, J. N., and DeLeo, J. A., 2001, “Quantification of Neural Tissue Injury in a Rat Radiculopathy Model: Comparison of Local Deformation, Behavioral Outcomes, and Spinal Cytokine mRNA for Two Surgeons,” Journal of Neuroscience Methods, 111, pp. 49-57.

[15] Winkelstein, B. A., Weinstein, J. N., and DeLeo, J. A., 2002, "The Role of Mechanical Deformation in Lumbar Radiculopathy: An in vivo Model," Spine, 27, pp. 27-33.

[16] Hunt, J., Fraser, R., Weinstein, J., DeLeo, J., 2000, “A new model of lumbar radiculopathy: validation of the use of the chromic gut model to study the pathophysiology of radicular pain," Proceedings of the International Society for the Study of the Lumbar Spine Annual Conference, Adelaide, Australia, Abstract \#43.

[17] Colburn, R., Rickman, A., and DeLeo, J., 1999, "The Effect of Site and Type of Nerve Injury on Spinal Glial Activation and Neuropathic Pain Behavior,” Experimental Neurology, 157, pp. 289-304. 
[18] Pedowitz, R., Garfin, S., Massie, J., Hargens, A., Swenson M., Myers, R., and Rydevik, B., 1992, "Effects of Magnitude and Duration of Compression on Spinal Nerve Root Conduction," Spine, 17, pp. 194-199.

[19] Fung, Y.C., 1965, Foundations of Solid Mechanics, Prentice-Hall, Inc.: Englewood Cliffs, NJ.

[20] DeLeo, J. A., and Colburn, R. W., 1996, "The Role of Cytokines in Nociception and Chronic Pain," Low Back Pain: A Scientific and Clinical Overview, Weinstein, J.N., Gordon, S.L. eds., AAOS Publishers, Rosemont, IL, pp. 163-185.

[21] Bain, A. C., Raghupathi, R., and Meaney, D. F., 2001, "Dynamic Stretch Correlates to Both Morphological Abnormalities and Electrophysiological Impairment in a Model of Traumatic Axonal Injury," Journal of Neurotrauma, 18, pp. 499-511.

[22] Olmarker, K., Rydevik, B., and Nordborg, C., 1993, “Autologous Nucleus Pulposus Induces Neurophysiologic and Histologic Changes in Porcine Cauda Equina Nerve Roots,” Spine, 18, pp. 1425-1432.

[23] Ozaktay, A. C., Cavanaugh, J. M., Asik, I., DeLeo, J. A., and Weinstein, J. N., 2002, "Dorsal root sensitivity to interleukin-1 beta, interleukin-6 and tumor necrosis factor in rats," European Spine Journal, 11, pp. 267-475. 
[24] Winkelstein, B. A., and DeLeo, J. A., 2002, "Nerve Root Tissue Injury Severity Differentially Modulates Spinal Glial Activation in a Rat Lumbar Radiculopathy Model: Considerations for Persistent Pain,” Brain Research, 956, pp. 294-301.

[25] Winkelstein, B. A., Finsness, E. D., Ridgway, A. B., DeLeo, J. A., 2002, “Anatomic and Biomechanical Considerations for Painful Lumbar Radiculopathy Models.” Society for Neuroscience $32^{\text {nd }}$ Annual Meeting, \#758.7.

[26] Inufusa, A., An, H. S., Lim, T., Hasegawa, T., Haughton, V. M., Nowicki, B. H., 1996, “Anatomic Changes of the Spinal Canal and Intervertebral Foramen Associated with FlexionExtension Movement," Spine, 21, pp. 2412-2420.

[27] Fujiwara, A., An, H.S., Lim, T., Haughton, V. M., 2001, "Morphologic Changes in the Lumbar Intervertebral Foramen Due to Flexion-Extension, Lateral Bending, and Axial Rotation,” Spine, 26, pp. 876-882.

[28] Schmid, M. R., Stucki, G., Duewell, S., Wildermuth, S., Romanowski, B., Hodler, J., 1999, "Changes in Cross-Sectional Measurements of the Spinal Canal and Intervertebral Foramina as a Function of Body Position,” Americal Journal of Radiology, 172, pp. 1095-1102.

[29] DeLeo, J. A., and Yezierski, R. P., 2001, "The Role of Neuroinflammation and Neuroimmune Activation in Persistent Pain," Pain, 90, pp. 1-6. 
[30] Winkelstein, B. A., Rutkowski, M. D., Sweitzer, S. M., Pahl, J. L., and DeLeo, J. A., 2001, "Nerve Injury Proximal or Distal to the DRG Induces Similar Spinal Glial Activation and Selective Cytokine Expression but Differential Behavioral Responses to Pharmacological Treatment," Journal of Comparative Neurology, 438, pp. 127-139.

[31] Maves, T. J., Pechman, P. S., Gebhart, G. F., and Meller, S. T., 1993, "Possible Chemical Contribution from Chromic Gut Sutures Produces Disorders of Pain Sensation Like Those Seen in Man," Pain, 54, pp. 57-69. 


\section{FIGURE LEGENDS}

Figure 1. The upper set of in vivo images on the left shows the initial unligated (reference) configuration (left) and the nerve root immediately following ligation (right). Superimposed on each image are the set of digitized boundary points along the nerve root (white dots) and the bony positional markers (black dots). The corresponding curve fits for the digitized nerve root boundaries are also shown in the set of images below them. The schematic on the right illustrates the geometric definitions for calculating radial strains along the nerve root. The radial strain magnitudes reported in this work were calculated in the region of maximal compression, indicated in this figure by the "ligation" region.

Figure 2. Mean mechanical sensitivity for all injury groups (low, tight, chromic, sham, and chromic exposure) of animals. Foot lift response frequency to stimulation with 12 gm von Frey filament is depicted over the 14 days of the study. There was a significant $(\mathrm{p}<0.0005)$ increase in mean mechanical allodynia for the tight and chromic ligation animals compared to both sham groups and the low silk ligation animals. Of note is the finding that while the allodynia of the tight and chromic animals were not significantly different, the applied nerve root strain in the tight group was significantly higher $(p=0.001)$ than that applied in the chromic ligation animals. The total number of responses resulting from 30 stimulations per animal was recorded and the group average and standard error are reported here. Behavioral responses for 2 gm von Frey filament testing were similar and are not shown here.

Figure 3. The overall mechanical sensitivity shows no significant correlation with the applied ligation strain in the presence of inflammatory chromic gut material. Overall mechanical 
sensitivity is measured by total number of paw withdrawals when tested using a 12 gram von Frey filament. The correlation coefficient (r) of this relationship is 0.151 .

Figure 4 In contrast to the chromic ligations, overall mechanical sensitivity is significantly correlated with the applied ligation strain in the absence of inflammatory material. Overall mechanical sensitivity is measured by total number of paw withdrawals when tested using a 12 gram von Frey filament. The correlation coefficient (r) of this relationship is 0.776 and is significant at $\mathrm{p}<0.05$.

Figure 5. This plot demonstrates the logistic regression analysis of mechanical tissue strains required to produce persistent pain in this ligation model. Each ligation injury was given a pain score of 1 or 0 based on the existence (1) or absence (0) of persistent mechanical allodynia over 14 days. Score were potted versus the applied ligation strain. This analysis predicted the $50^{\text {th }}$ percentile strain of $20.8 \%$ applied strain and the $95^{\text {th }}$ percentile threshold for pain persistence at a strain of $22.2 \%$. These thresholds are indicated by dotted and straight lines on this plot. Individual animal responses are shown as squares. Thresholds for pain initiation were determined using similar approaches (not shown). 

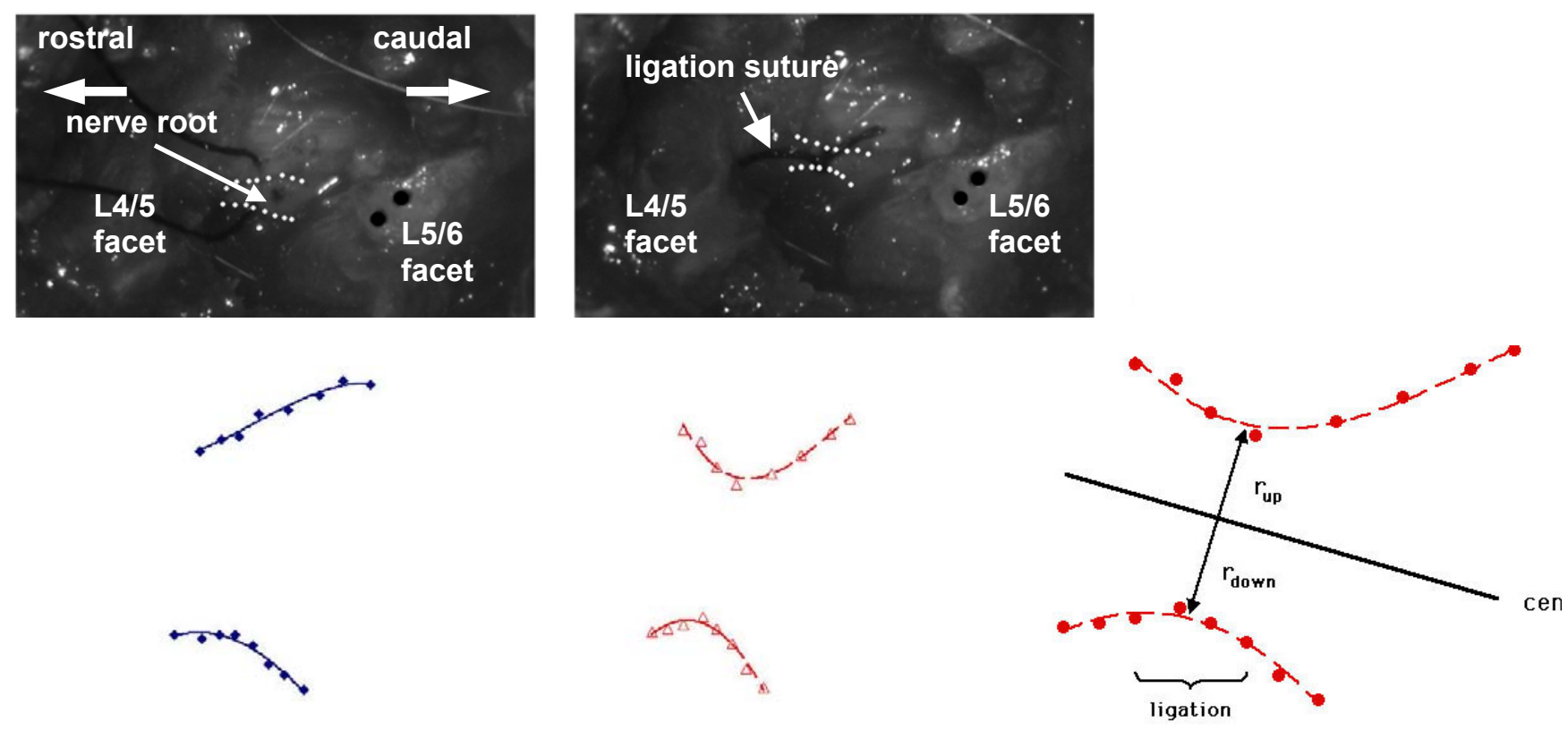

boundaries of reference configuration

boundaries immediately

following ligation 


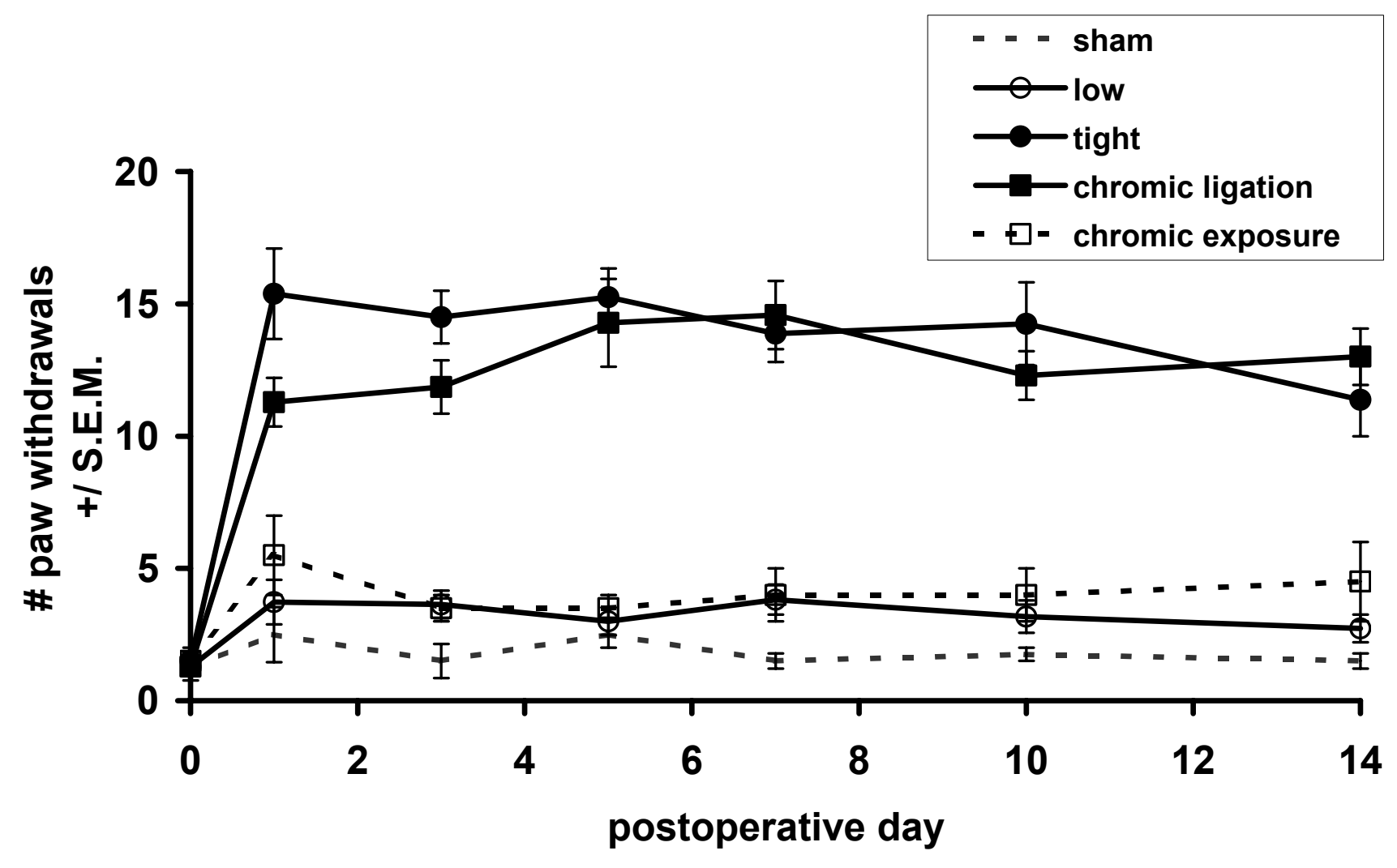




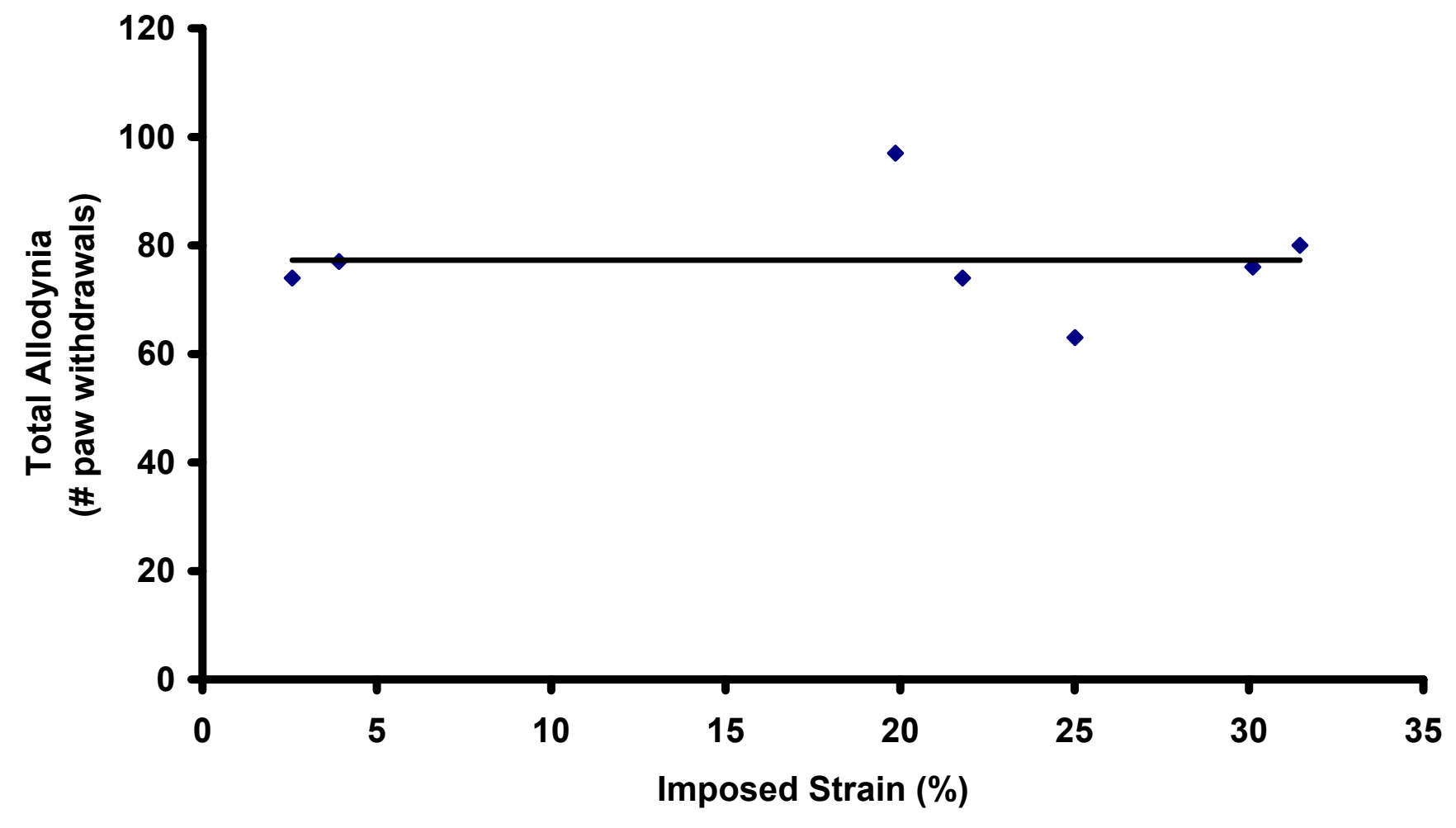




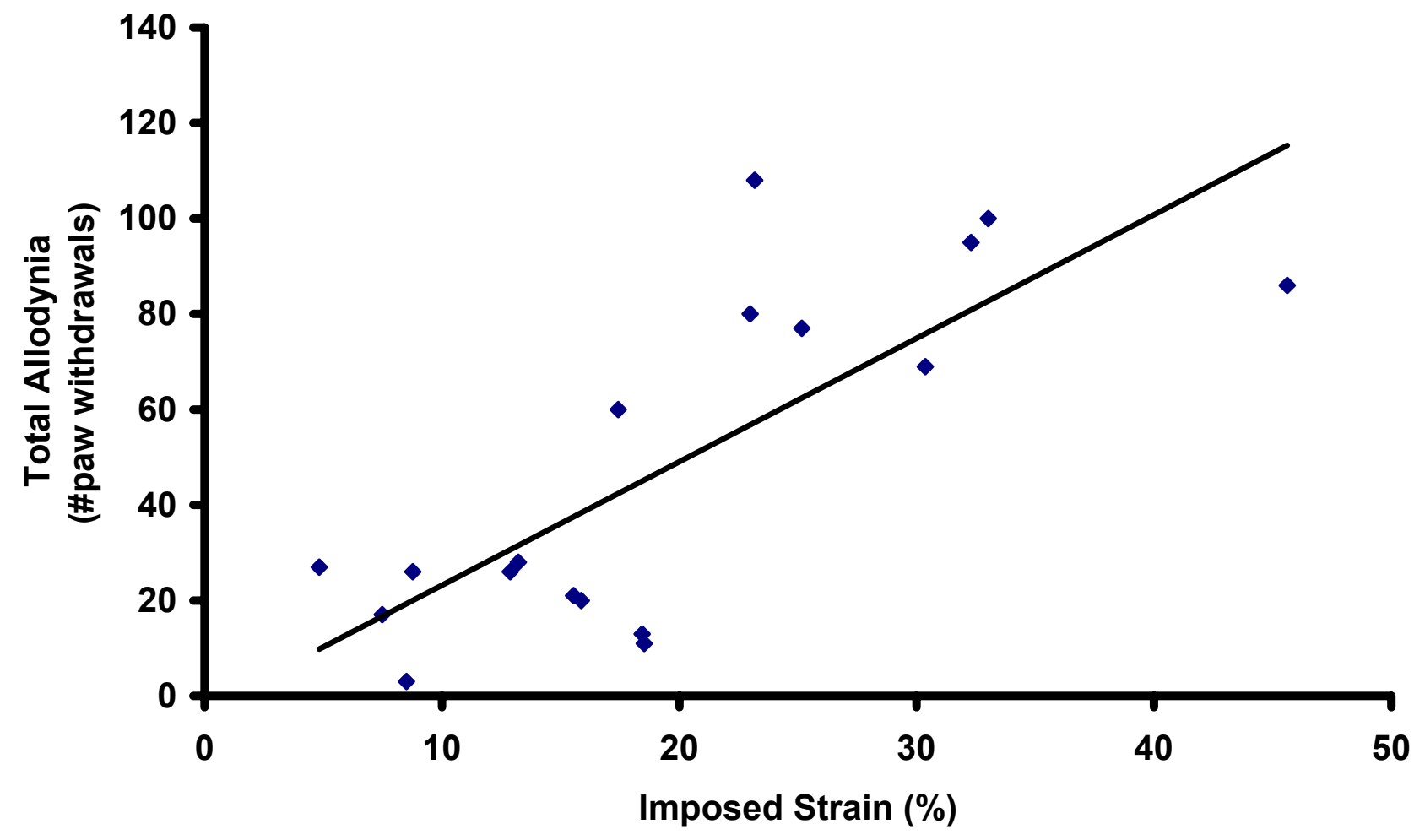




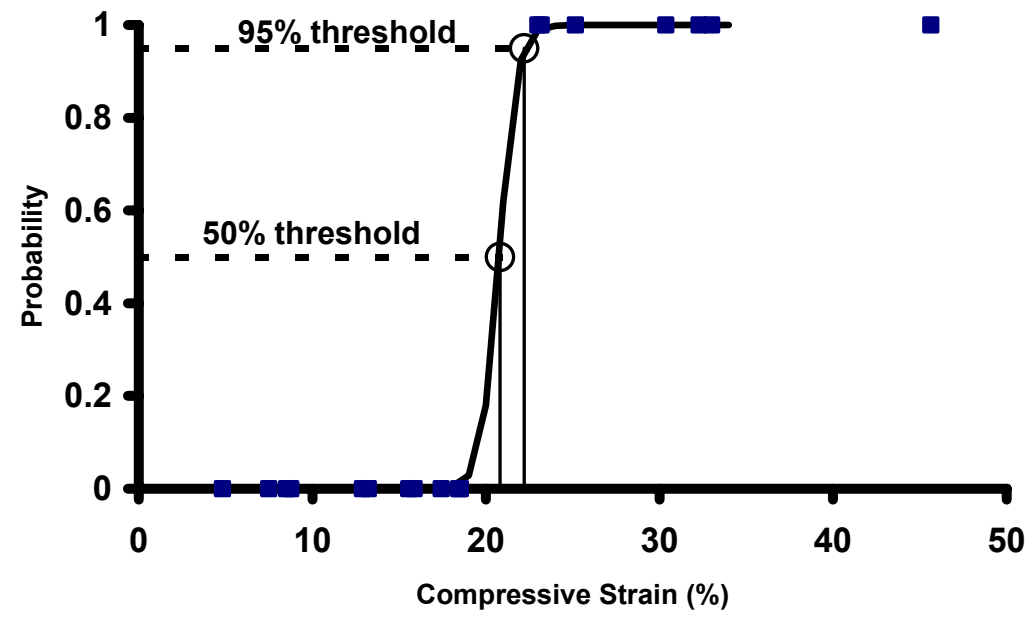


Table 1. Correlation Coefficients for Imposed

Injury Strain Magnitude \& Chemical Insult Nociceptive Silk

Chromic

Measure

Ligation

Ligation

Paw Withdrawals

(2 gram stimulation)

$0.810 *$

0.045

Paw Withdrawals

(12 gram stimulation)

$0.776 *$

0.151

* Correlation is significant $(\mathrm{p}<0.05)$.

All correlations are positive. 